\title{
EDITORIAL
}

\section{Right ventricular ejection fraction and NT-proBNP are both indicators of wall stress in pulmonary} hypertension

\author{
A. Vonk Noordegraaf* and N. Westerhof*,\#
}

$\Delta$ lthough many therapeutic options have become available in recent years for the treatment of pulmonary arterial hypertension $(\mathrm{PAH})$, there is still no cure available. The primary cause of death is right ventricular failure. Therefore, monitoring right ventricular function is a way to guide therapeutic decisions and a logical step forward to improve life expectancy in PAH patients. Although magnetic resonance imaging (MRI) is considered the gold standard in measuring right ventricular function, the technique is expensive with limited accessibility; therefore, it is unlikely to be frequently used in PAH centres on a regular basis. Therefore, technically simpler methods are welcomed.

In current issue of the European Respiratory Journal, BLYTH et al. [1] report that N-terminal B-type natriuretic peptide (NTproBNP) might act as a simple and effective alternative to MRI measurement. Thus, the results of BLYTH et al. [1] confirm those of earlier studies suggesting a relationship between cardiac function in PAH and NT-proBNP [2,3]. However, the question remains how to explain this relationship. BLYTH et al. [1] also showed that NT-proBNP correlates inversely with the right ventricular ejection fraction (RVEF), which is assumed to be a measure of right ventricular systolic function. These relationships can be explained as follows.

Previous studies carried out in isolated papillary muscle, in animal models and in patients with aorta stenosis revealed that wall stress modulates BNP gene expression in the cardiomyocyte [4-6]. Although all of these studies were performed in the left ventricle, there are no arguments to suggest that the right ventricular myocytes would behave differently. Wall stress is related to pressure and ventricular volume, and is inversely proportional to ventricular wall volume [7]. Since two previous studies showed that systolic pressure and wall volume are closely related to each other in pulmonary hypertension [8,9], their combined contribution may be neglected, making ventricular volume the most important determinant of wall

*Depts of Pulmonary Diseases and \#Physiology, ICaR-Vu, VU University Medical Center, Amsterdam, the Netherlands.

STATEMENT OF INTEREST: None declared

CORRESPONDENCE: A. Vonk Noordegraaf, Dept of Pulmonary Diseases, VU University Medical Center, P0 Box 7057, 1007 MB Amsterdam, the Netherlands. Fax: 31 204444328. E-mail: a.vonk@vumc.nl stress. A higher end-diastolic volume corresponds to more stress and is a main determinant of diastolic wall stress. In addition, systolic stress is lower with increased stroke volume [10]. Therefore, stress can be increased by either an increase in end-diastolic volume or a reduction of stroke volume, both of which lead to a reduction in RVEF. This finding is in agreement with QUAIFE et al. [11] who found that RVEF and wall stress are closely and inversely related to each other $(\mathrm{r}=0.80)$. Therefore, RVEF should be considered as a reflection of right ventricular wall stress rather then as a parameter of right ventricular systolic function.

Elevated levels of NT-proBNP reflect increased wall stress and, therefore, a maladaptation of the right ventricle trying to cope with the increased afterload. In this respect, both a high NTproBNP level and a low RVEF should be interpreted as an inadequate adaptation of the right ventricle to pump against a disproportionately increased right ventricular afterload.

NT-proBNP is a good marker by which to monitor pulmonary hypertension, since it answers the question of whether the right ventricle is well adapted to the increased afterload. Reduction of NT-proBNP to a certain level can serve as a guide in the treatment of pulmonary hypertension and is of special interest as a primary end-point in the study of a stepwise approach using combination therapies to reach this goal. BLYTH et al. [1] showed that NT-proBNP levels $>1,600 \mathrm{ng} \cdot \mathrm{L}^{-1}$ are associated with an RVEF $<42 \%$. Interestingly, FIJALKOWSKA et al. [12] showed that a similar NT-proBNP level was found to be useful in identifying patients with poor long-term prognosis.

If wall stress is indeed the main determinant of NT-proBNP, lowering NT-proBNP levels can be achieved in two different ways: 1) by reduction of right ventricular afterload; or 2) by a better adaptation of the right ventricle. Although much attention has been paid in recent years to treating the underlying disease and, therefore, reducing afterload, treatment failure is still frequent. In contrast, improving right ventricular adaptation has received little attention, and no treatment exists as yet to improve this adaptation. Despite this observation, in patients with congenital heart disease the right ventricle is capable of handling high pressures if adequately adapted.

The novel insights obtained from the study by BLYTH et al. [1] not only provide the possibility of obtaining insight into the mechanisms of right ventricular adaptation and the 
subsequent search for therapeutic interventions, but they also may suggest how to improve this process. Thus, the study by BLYTH et al. [1] is an important step forward in the treatment of pulmonary hypertension and provides the measures of how to do so.

\section{REFERENCES}

1 Blyth KG, Groenning BA, Mark PB, et al. NT-proBNP can be used to detect right ventricular systolic dysfunction in pulmonary hypertension. Eur Respir J 2007; 29: 737-744.

2 Nagaya N, Nishikimi T, Okano Y, et al. Plasma brain natriuretic peptide levels increase in proportion to the extent of right ventricular dysfunction in pulmonary hypertension. J Am Coll Cardiol 1998; 31: 202-208.

3 Gan CT, McCann GP, Marcus JT, et al. NT-proBNP reflects right ventricular structure and function in pulmonary hypertension. Eur Respir J 2006; 28: 1190-1194.

4 Wiese S, Breyer T, Dragu A, et al. Gene expression of brain natriuretic peptide in isolated atrial and ventricular human myocardium: influence of angiotensin II and diastolic fiber length. Circulation 2000; 102: 3074-3079.

5 Nakagawa O, Ogawa Y, Itoh $\mathrm{H}$, et al. Rapid transcriptional activation and early mRNA turnover of brain natriuretic peptide in cardiocyte hypertrophy: evidence for brain natriuretic peptide as an "emergency" cardiac hormone against ventricular overload. J Clin Invest 1995; 96: 1280-1287.
6 Vanderheyden M, Goethals M, Verstreken S, et al. Wall stress modulates brain natriuretic peptide production in pressure overload cardiomyopathy. J Am Coll Cardiol 2004; 44: 2349-2354.

7 Arts T, Bovendeerd PH, Prinzen FW, Reneman RS. Relation between left ventricular cavity pressure and volume and systolic fiber stress and strain in the wall. Biophys J 1991; 59: 93-102.

8 Saba TS, Foster J, Cockburn M, Cowan M, Peacock AJ. Ventricular mass index using magnetic resonance imaging accurately estimates pulmonary artery pressure. Eur Respir J 2002; 20: 1519-1524.

9 Roeleveld RJ, Marcus JT, Boonstra A, et al. A comparison of noninvasive MRI-based methods of estimating pulmonary artery pressure in pulmonary hypertension. J Magn Reson Imaging 2005; 22: 67-72.

10 Weber KT, Janicki JS. Myocardial oxygen consumption: the role of wall force and shortening. Am J Physiol 1977; 233: H421-H430.

11 Quaife RA, Chen MY, Lynch D, et al. Importance of right ventricular end-systolic regional wall stress in idiopathic pulmonary arterial hypertension: a new method for estimation of right ventricular wall stress. Eur J Med Res 2006; 11: 214-220.

12 Fijalkowska A, Kurzyna M, Torbicki A, et al. Serum Nterminal brain natriuretic peptide as a prognostic parameter in patients with pulmonary hypertension. Chest 2006; 129: 1313-1321. 